\begin{tabular}{|c|l|}
\hline Title & $\begin{array}{l}\text { Susceptibility-Weighted Magnetic Resonance Imaging Detects Impaired Cerebral Hemodynamics in the Superior } \\
\text { Sagittal Sinus Thrombosis : C ase Report }\end{array}$ \\
\hline Author(s) & $\begin{array}{l}\text { Kawabori, Masahito; Kuroda, Satoshi; Kudo, Kohsuke; Terae, Satoshi; Kaneda, Makoto; Nakay ama, Naoki; I wasaki, } \\
\text { Yoshinobu }\end{array}$ \\
\hline Citation & $\begin{array}{l}\text { Neurologia medico-chirurgica, 49(6), 248-251 } \\
\text { https://doi.org/40.2176/hmc.49.248 }\end{array}$ \\
\hline Issue Date & 2009-06 \\
\hline Doc URL & http://hdl.handle.net/2115/70774 \\
\hline Rights(URL) & https://reativecommons.org/icenses/by-nc-nd/4.0/ \\
\hline Type & article \\
\hline File Information & E8_49_248.pdf \\
\hline
\end{tabular}

Instructions for use 


\title{
Susceptibility-Weighted Magnetic Resonance Imaging Detects Impaired Cerebral Hemodynamics in the Superior Sagittal Sinus Thrombosis
}

\author{
-Case Report- \\ Masahito Kawabori, Satoshi Kuroda, Kohsuke Kudo*, Satoshi TeraE**, \\ Makoto KANEDA***, Naoki NAKAYAMA, and Yoshinobu IWASAKI \\ Departments of Neurosurgery, ${ }^{* *}$ Radiology, and ${ }^{* * *}$ Pediatrics, \\ Hokkaido University Graduate School of Medicine, Sapporo, Hokkaido; \\ *Department of Radiology, Iwate Medical University, Morioka, Iwate
}

\begin{abstract}
An 11-year-old female receiving treatment for acute lymphoblastic leukemia presented with superior sagittal sinus (SSS) thrombosis. $T_{1}$-weighted, $T_{2}$-weighted, and fluid-attenuated inversion recovery magnetic resonance (MR) imaging, and MR venography showed that the SSS was totally occluded by thrombus. Susceptibility-weighted MR imaging showed hypointense thrombus in the SSS and markedly dilated cortical veins over the bilateral cerebral hemispheres. Two days later, her symptoms had slightly resolved. Iodine-123 $\mathrm{N}$-isopropyl-p-iodoamphetamine single photon emission computed tomography showed marked decrease of cerebral blood flow in the bilateral frontal lobes, indicating that venous congestion had disturbed the cerebral hemodynamics. MR venography showed that the SSS was still mostly occluded, but susceptibility-weighted imaging showed that the dilation of the cortical veins was less marked, suggesting that collateral venous routes had gradually developed. The finding of dilated cortical veins had almost disappeared at 28 days after the onset. Susceptibility-weighted imaging can be used as a non-invasive method to monitor the severity of venous congestion caused by cerebral venous sinus thrombosis.
\end{abstract}

Key words: susceptibility-weighted magnetic resonance imaging, superior sagittal sinus thrombosis, cerebral hemodynamics

\section{Introduction}

Cerebral venous sinus thrombosis (CVST) is an uncommon disease with potentially serious consequences. ${ }^{1,4,5,8,10,11)}$ The diagnosis of CVST can be most easily established based on the findings of magnetic resonance (MR) venography and $T_{1-}$ weighted, $\mathrm{T}_{2}^{*}$-weighted, $\mathrm{T}_{2}$-weighted, fluid-attenuated inversion recovery, and diffusion-weighted MR imaging. ${ }^{4,8,10)}$ CVST can also be identified by computed tomography venography and conventional angiography, but MR imaging can directly detect thrombus without requiring contrast medium administration, and MR venography can be performed at the same examination. However, MR venography using phase contrast or time-of-flight methods may misinterpret conditions such as hypoplastic trans-

Received May 28, 2008; Accepted January 30, 2009 verse sinus or physiological retrograde flow $^{6)}$ as sinus occlusion. Furthermore, MR venography cannot establish the diagnosis of isolated cortical venous thrombosis. ${ }^{4)}$

Susceptibility-weighted MR imaging is an extremely sensitive technique to detect magnetic susceptibility changes between blood product and tissues, ${ }^{1)}$ and was originally developed as a method of high resolution MR venography based on the blood oxygen level dependent effect. ${ }^{1,3,7,9,12,13)}$ Susceptibility-weighted MR imaging shows intravascular venous deoxygenated blood as low signal intensity, so intracranial venous structures that contain deoxygenated blood appear dark, and is also extremely sensitive to extravascular blood products as well as the presence of other substances such as iron, some forms of calcification, and air. Susceptibility-weighted MR imaging is useful for the detection of hemorrhagic lesions, vascular malformations such as 
capillary telangiectasia, demonstration of venous thrombosis, and calcium or iron deposition in neurodegenerative disorders, ${ }^{12-14)}$ and may demonstrate increased oxygen extraction in areas of brain infarction or hypoxemia. ${ }^{13)}$ Susceptibility-weighted MR imaging is expected to become a diagnostic method for CVST because of the high sensitivity to detect clots and to delineate small cerebral veins, ${ }^{9,12,13)}$ but the usefulness to detect CVST-related disturbance of cerebral hemodynamics is unclear.

Here we present a case of CVST in which serial susceptibility-weighted MR imaging provided important information on the sequential changes in cerebral hemodynamics.

\section{Case Report}

An 11-year-old female presented with acute lymphoblastic leukemia, and underwent chemotherapy using vincristine, cyclophosphamide, pirarubicin, and L-asparaginase. Her disease showed remission after induction chemotherapy, but she complained of unusual headache 4 weeks later. She complained of severe headache and numbness of her left upper extremity on the next day, and then developed generalized seizure.

Emergent $\mathrm{T}_{1}$ - and $\mathrm{T}_{2}$-weighted $\mathrm{MR}$ imaging, and MR venography (Signa Horizon 1.5T; GE Yokokawa Medical System, Tokyo) showed that the superior sagittal sinus (SSS) was totally occluded by thrombus (Fig. 1A, B, D). Diffusion-weighted MR imaging demonstrated a hyperintense area in the left parietal lobe with decreased apparent diffusion coefficient (Fig. 1C). Susceptibility-weighted MR imaging also showed hypointense thrombus in the SSS, and markedly dilated cortical veins over the bilateral cerebral hemispheres (Fig. 1E, F). The diagnosis was SSS thrombosis associated with venous infarction in the left parietal lobe. She was immediately treated with heparin and anticonvulsant agent.

Two days later, her symptoms had slightly resolved. Iodine-123 N-isopropyl-p-iodoamphetamine single photon emission computed tomography (SPECT) showed marked decrease of cerebral blood flow (CBF) in the bilateral frontal lobes (Fig. 1G), indicating that venous congestion had disturbed the cerebral hemodynamics in the frontal lobes. MR venography showed that the SSS was still mostly occluded, but susceptibility-weighted MR imaging indicated that the dilation of the cortical veins had become less marked (Fig. 1H, I). Her symptoms had almost resolved at 4 days after the onset, strongly suggesting that collateral venous routes had gradually developed. Twenty days after the onset, ${ }^{15} \mathrm{O}$ positron emission tomography (PET) showed nor- malized CBF in the bilateral frontal lobes (Fig. 1J). MR venography showed that the SSS had mostly reopened, and susceptibility-weighted MR imaging indicated that dilation of the cortical veins had disappeared by 28 days after the onset (Fig. 1K, L). She received oral warfarin. Follow-up MR imaging 2 months after the onset showed old venous infarction in the left parietal cortex (Fig. 1M). She was discharged from hospital without neurological deficit. She has experienced no further episode of CVST.

Our method of susceptibility-weighted MR imaging used the three-dimensional fast low-angle shot sequence with imaging parameters $49 / 40 \mathrm{msec}$ repetition time/echo time, $20^{\circ}$ flip angle, 1 excitation, and $80 \mathrm{~Hz} /$ pixel bandwidth. Forty-four $3.25 \mathrm{~mm}$ thick slices were acquired with a $240 \times 188.6 \mathrm{~mm}$ field of view and a matrix size of $448 \times 314$. The parallel imaging technique was used and the acquisition time was 5 minutes 53 seconds. Susceptibilityweighted images were created using magnitude and phase images. A phase mask was created by setting all negative-phase values to unity, and normalizing the positive-phase values ranging from $0^{\circ}$ to $180^{\circ}$ to a linear gray scale of values from 1 to 0 , respectively. This normalized phase mask was multiplied four times against the original magnitude image. Finally, a minimum intensity projection over 2 sections was performed to display the processed data as contiguous $6.5-\mathrm{mm}$ thick sections in the transverse plane. ${ }^{3)}$

\section{Discussion}

In the present case, susceptibility-weighted MR imaging demonstrated marked hypointense clots in the SSS and dilated, hypointense cortical veins, supporting the usefulness for the diagnosis of CVST. ${ }^{13)}$ Serial studies confirmed that the dilated cortical veins gradually normalized after treatment. Susceptibilityweighted MR imaging can be helpful for demonstrating unsuspected thrombosis with a much better resolution than conventional imaging, and can also demonstrate parenchymal brain hemorrhage occurring after venous thrombosis more sensitively than other MR imaging sequences. ${ }^{13)}$ Susceptibilityweighted MR imaging provides both phase and magnitude information, so facilitates the detection of cortical venous thrombosis. ${ }^{12)}$ High-resolution oxygen-level dependent venography, which is the same technique, can be used to show sinus and dural venous thrombosis, and major cortical draining veins. ${ }^{9)}$ Susceptibility-weighted MR imaging may also be useful in monitoring the effectiveness of thrombolytic therapy. ${ }^{9)}$

Interestingly, the dilated cortical veins started to normalize when the SSS was still mostly occluded in 

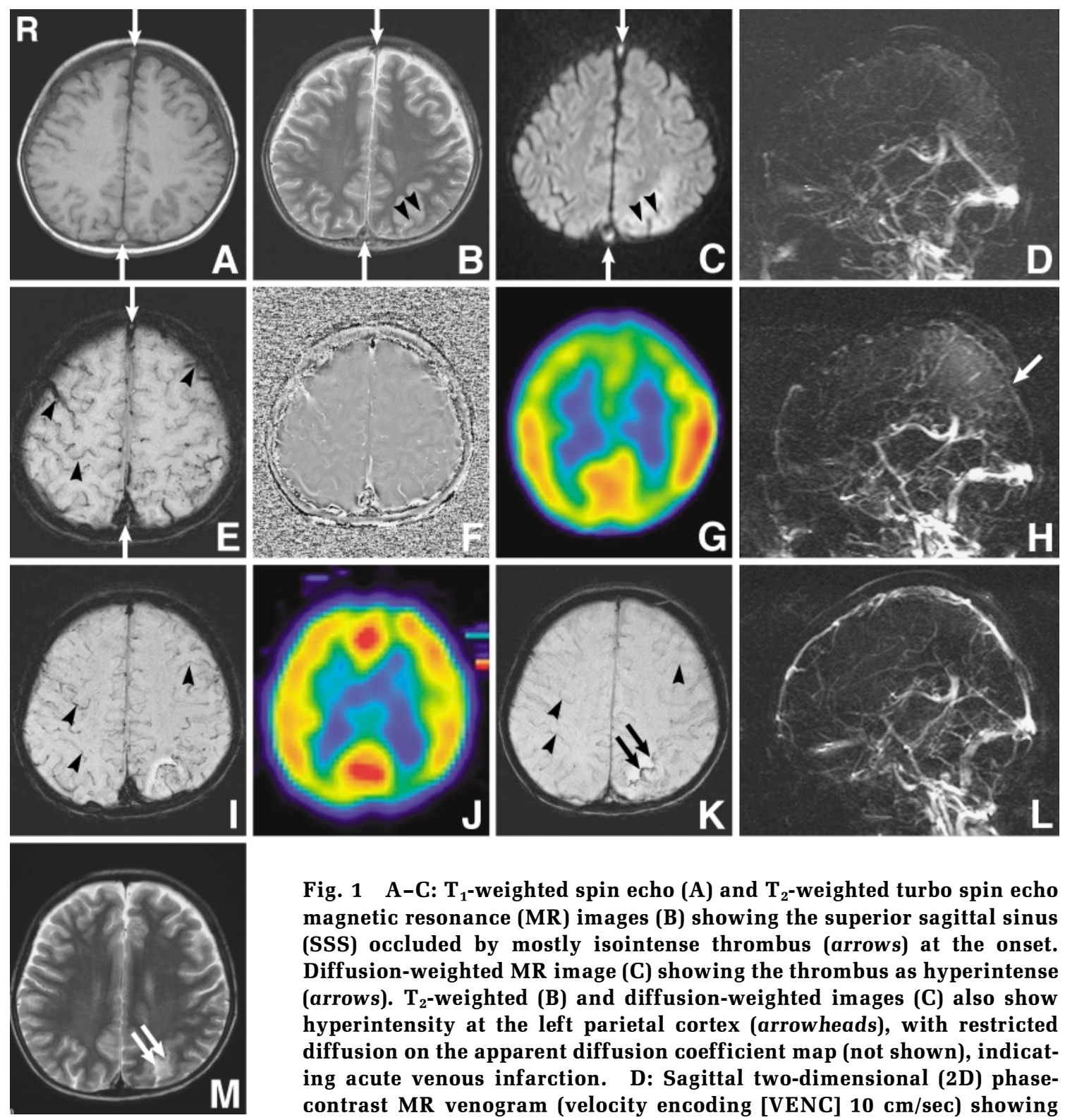

Fig. 1 A-C: $T_{1}$-weighted spin echo (A) and $T_{2}$-weighted turbo spin echo magnetic resonance (MR) images $(B)$ showing the superior sagittal sinus (SSS) occluded by mostly isointense thrombus (arrows) at the onset. Diffusion-weighted MR image (C) showing the thrombus as hyperintense (arrows). $\mathrm{T}_{2}$-weighted (B) and diffusion-weighted images (C) also show hyperintensity at the left parietal cortex (arrowheads), with restricted diffusion on the apparent diffusion coefficient map (not shown), indicating acute venous infarction. D: Sagittal two-dimensional (2D) phasecontrast MR venogram (velocity encoding [VENC] $10 \mathrm{~cm} / \mathrm{sec}$ ) showing total occlusion of the SSS, and patent cortical veins. E: Susceptibilityweighted MR image with minimum intensity projection showing the thrombus as mostly markedly hypointense (arrowheads), and the SSS as dark and dilated (arrows). F: Susceptibility-weighted phase-contrast MR image showing that positive phases in the cortical veins, indicating deoxygenated blood within the patent veins. G: Iodine-123 $\mathrm{N}$-isopropyl-p-iodoamphetamine single photon emission computed tomography scan showing marked decrease of cerebral blood flow (CBF) in the bilateral frontal lobes 2 days after the onset. H: Sagittal 2D phase-contrast MR venogram (VENC 10 $\mathrm{cm} / \mathrm{sec}$ ) showing the SSS is mostly occluded, but the posterior part is partially reopened (arrow). I: Susceptibility-weighted MR image with minimum intensity projection showing reduced but still mild dilation of the cortical veins (arrowheads), and a hyperintense area and multiple dotty hypointense areas in the left parietal lobe indicating infarct and microbleeds, respectively. $\mathrm{J}:{ }^{15} \mathrm{O}$ positron emission tomography scan showing normal CBF 20 days after the onset. K: Susceptibility-weighted MR image with minimum intensity projection showing the normal superior cerebral veins (arrowheads) 28 days after the onset, and a linear hypointense area along the left parietal cortex considered to be hemosiderin deposition at the infarct (double arrows). L: Sagittal $2 D$ phase-contrast MR venogram (VENC $10 \mathrm{~cm} / \mathrm{sec}$ ) showing that the SSS is mostly reopened. M: $\mathrm{T}_{2}$-weighted MR image 2 months after the onset showing the old venous infarction in the left parietal cortex (double arrows). 
the present case, which may indicate that the venous congestion gradually improved with the development of collateral circulation. Diffusion-weighted imaging, ${ }^{8)}$ SPECT ${ }^{2)}$ and PET ${ }^{5)}$ are also useful to assess the pathophysiological aspects of CVST, but the treatment efficacy is difficult to evaluate with these imaging techniques. In contrast, serial susceptibility-weighted MR imaging is a non-invasive method to monitor the severity of venous congestion caused by CVST.

Susceptibility-weighted MR imaging has a few limitations. First, small venous structures are difficult to differentiate from small hemorrhage and thrombosis, because the signal characteristics are similar, but discrimination may be possible by using phase information. ${ }^{12)}$ Second, the relatively long acquisition time of up to 10 minutes leads to a relatively high probability of motion artifacts. Third, any hypointense cortical veins do not really represent the true vascular diameter, and the venous caliber might be overestimated because of the susceptibility effect caused by higher concentrations of intravascular deoxyhemoglobin associated with venous stasis. Further prospective studies are required to confirm the impact of susceptibilityweighted MR imaging as a diagnostic tool for venous congestion caused by CVST in a larger patient population.

The present case illustrates that susceptibilityweighted MR imaging can demonstrate congestion of the cortical veins and sequential changes. Susceptibility-weighted MR imaging is a non-invasive and useful method to monitor the severity of venous congestion by assessing the status of the cortical veins, and can provide additional value to conventional MR imaging and MR venography.

\section{References}

1) Deistung A, Mentzel HJ, Rauscher A, Witoszynskyj S, Kaiser WA, Reichenbach JR: Demonstration of paramagnetic and diamagnetic cerebral lesions by using susceptibility weighted phase imaging (SWI). Z Med Phys 16: 261-267, 2006

2) Front D, Israel O, Even-Sapir E, Feinsud M: Superior sagittal sinus thrombosis: assessment with Tc-99m labeled red blood cells. Radiology 158: 453-456, 1986

3) Haacke EM, Xu Y, Cheng YC, Reichenbach JR: Susceptibility weighted imaging (SWI). Magn Reson Med 52: 612-618, 2004

4) Idbaih A, Boukobza M, Crassard I, Porcher R, Bousser MG, Chabriat H: MRI of clot in cerebral venous thrombosis: high diagnostic value of susceptibilityweighted images. Stroke 37: 991-995, 2006

5) Kawai N, Shindou A, Masada T, Tamiya T, Nagao S: Hemodynamic and metabolic changes in a patient with cerebral venous sinus thrombosis: evaluation using O-15 positron emission tomography. Clin Nucl Med 30: 391-394, 2005

6) Kudo K, Terae S, Ishii A, Omatsu T, Asano T, Tha KK, Miyasaka K: Physiologic change in flow velocity and direction of dural venous sinuses with respiration: MR venography and flow analysis. AJNR Am J Neuroradiol 25: 551-557, 2004

7) Lee BC, Vo KD, Kido DK, Mukherjee P, Reichenbach J, Lin W, Yoon MS, Haacke M: MR high-resolution blood oxygenation level-dependent venography of occult (low-flow) vascular lesions. AJNR Am J Neuroradiol 20: 1239-1242, 1999

8) Mullins ME, Grant PE, Wang B, Gonzalez RG, Schaefer PW: Parenchymal abnormalities associated with cerebral venous sinus thrombosis: assessment with diffusion-weighted MR imaging. AJNR Am J Neuroradiol 25: 1666-1675, 2004

9) Reichenbach JR, Jonetz-Mentzel L, Fitzek C, Haacke EM, Kido DK, Lee BC, Kaiser WA: High-resolution blood oxygen-level dependent MR venography (HRBV): a new technique. Neuroradiology 43: 364369,2001

10) Sajjad Z: MRI and MRV in cerebral venous thrombosis. J Pak Med Assoc 56: 523-526, 2006

11) Selim M, Fink J, Linfante I, Kumar S, Schlaug G, Caplan LR: Diagnosis of cerebral venous thrombosis with echo-planar T2*-weighted magnetic resonance imaging. Arch Neurol 59: 1021-1026, 2002

12) Thomas B, Somasundaram S, Thamburaj K, Kesavadas C, Gupta AK, Bodhey NK, Kapilamoorthy TR: Clinical applications of susceptibility weighted MR imaging of the brain - a pictorial review. Neuroradiology 50: 105-116, 2008

13) Tong KA, Ashwal S, Obenaus A, Nickerson JP, Kido D, Haacke EM: Susceptibility-weighted MR imaging: a review of clinical applications in children. AJNR Am J Neuroradiol 29: 9-17, 2008

14) Yoshida $Y$, Terae $S$, Kudo $K$, Tha KK, Imamura $M$, Miyasaka K: Capillary telangiectasia of the brain stem diagnosed by susceptibility-weighted imaging. J Comput Assist Tomogr 30: 980-982, 2006

Address reprint requests to: Masahito Kawabori, M.D., Department of Neurosurgery, Hokkaido University Graduate School of Medicine, North-15 West-7, Kita-ku, Sapporo 060-8638, Japan. e-mail: masahitokawabori@yahoo.co.jp 